\title{
Unitarity corrections to the structure functions through the dipole picture
}

\author{
M. B. Gay Ducati* and M. V. T. Machado ${ }^{\dagger}$ \\ Instituto de Física, Universidade Federal do Rio Grande do Sul, Caixa Postal 15051, CEP 91501-970, Porto Alegre, RS, Brazil
}

(Received 8 November 2001; published 24 June 2002)

\begin{abstract}
We study the dipole picture for the description of deep inelastic scattering, focusing on the structure functions which are driven directly by the gluon distribution. One performs estimates using the effective dipole cross section given by the Glauber-Mueller approach in QCD, which encodes the corrections due to the unitarity effects associated with the saturation phenomenon. We also address issues about frame invariance of the calculations when analyzing the observables.
\end{abstract}

DOI: 10.1103/PhysRevD.65.114019

PACS number(s): 12.38.Bx, 13.60.Hb

\section{INTRODUCTION}

The scattering experiments on deep inelastic electronproton (DIS) at the DESY ep collider HERA have provided measurements of the inclusive structure function $F_{2}\left(x, Q^{2}\right)$ as well as of the $F_{L}$ and $F_{2}^{c \bar{c}}$ in very small Bjorken variable $x$ values $\left(x \ll 10^{-2}\right)$. In these collisions the proton target is analyzed through a hard probe with virtuality $Q^{2}=-q^{2}$. The momentum fraction is $x \sim Q^{2} / 2 p \cdot q$, where $p$ and $q$ are the four-momenta of the incoming proton and of the virtual photon probe. In the kinematical region of small $x$, the gluon is the leading parton driving the behavior of the deep inelastic observables. The standard QCD evolution [1] gives a powerlike growth for the gluon distribution and related quantities, and this feature leads, in principle, to the unitarity violation at asymptotic energies, requiring control of the gluon distribution in high energies. In the partonic language, in the infinite momentum frame, the small momentum fraction region corresponds to the high parton density domain, which is connected with the black disk limit of the proton target and with the parton recombination phenomenon. These issues can be addressed through a nonlinear dynamics beyond the usual Dokshitzer-Gribov-Lipatov-Altarelli-Parisi (DGLAP) formalism (for a review, see [2]). A complete knowledge of the nonlinear dynamical regime plays an important role in the theoretical description of the reactions in the forthcoming experiments of the BNL Relativistic Heavy Ion Collider (RHIC) and CERN Large Hardron Collider (LHC).

In the Breit frame, the QCD factorization theorem allows us to calculate the scattering processes through the convolution of the partonic subprocess with the parton distribution functions (PDF's). There, the degrees of freedom of the theory are the quasifree partons (quarks and gluons). On the other hand, more recently the theoretical description of the small $x$ physics has been widely analyzed in the target rest frame, which is a powerful tool concerning a unified picture for both the inclusive and the diffractive scatterings, including vector meson production [3]. Now, the degrees of freedom are the color dipoles, which are the simplest configurations considering the virtual photon Fock state expansion. Its

\footnotetext{
*Email address: gay@if.ufrgs.br

${ }^{\dagger}$ Email address: magnus@if.ufrgs.br
}

main appeal is a quite simplified picture for the different mentioned processes, based on general properties of quantum mechanics.

The description of DIS in the color dipole picture is quite intuitive, allowing a simple representation instead of the involved one from the Breit (infinite momentum) frame, and such framework was proposed by Gribov many years ago [4]. Considering small values of the Bjorken variable $x$, the virtual photon fluctuates into a $q \bar{q}$ pair (dipole) with fixed transverse separation $r$ at large distances upstream of the target and interacts in a short time with the proton. More complicated configurations should be considered for larger transverse size systems, for instance the photon Fock state $q \bar{q}+$ gluon. An immediate consequence of the lifetime of the pair $\left(l_{c}=1 / 2 m_{p} x\right)$ to be bigger than the interaction's one is the factorization between the photon wave function and the cross section dipole proton in the $\gamma^{*} p$ total cross section. The wave functions are perturbatively calculable, namely through QED for the $q \bar{q}$ configuration [5] and from QCD for $q \bar{q} G$ [6]. The effective dipole cross section should be modeled and encloses both perturbative and nonperturbative content. However, since the interaction strength relies only on the configuration of the interacting system the dipole cross section turns out to be universal and may be employed in a wide variety of small $x$ processes [3].

Currently, there are several models for the dipole cross section based either in pure phenomenological parametrizations or in a more theoretical ground (for a review, see Ref. [3]). The main feature in those models is the description of the energy dependence of the interaction, namely taking into account the interplay between hard and soft domains. The dipole cross section should be consistent with the sharp growth on energy at small transverse separation $r$ (large gluon density) and a softer behavior for larger $r$ (Regge-like phenomenology). An additional ingredient is the expectation for saturation effects in high energies as a consequence of the unitarity requirements. Indeed, the growth of the gluon distribution should be tamed at very small $x$ and it has been found that the corrections are important already in the present HERA kinematics [7]. Moreover, such effects are associated with higher twist contributions concerning the standard linear evolution equation, i.e., the DGLAP formalism [8].

Here, we take into account a formalism providing the uni- 
tarity corrections to the deep inelastic scattering at small $x$, namely the Glauber-Mueller approach in QCD. It was introduced by Mueller [9], who developed the Glauber formalism to study saturation effects in the quark and gluon distributions in the nucleus considering the heavy onium scattering. Afterwards, the authors of Ref. [10] extended that approach that has as a limit the Gribov-Levin-Ryskin (GLR) results [11]. It is obtained as an evolution equation taking into account the unitarity corrections (perturbative shadowing), generating a nonlinear dynamics which is related with higher twist contributions. Its main characteristic is to provide a theoretical framework for the saturation effects, relying on the multiscattering of the perturbative QCD (PQCD) Pomeron. The latter is represented through the usual gluonic ladder in the double logarithmic approximation.

Summarizing the Glauber-Mueller approach, the gluonic content of the nucleus or nucleons is obtained in the following way: in the rest frame, a virtual probe (gluon) decays into a gluon pair interacting with the nucleon inside the nucleus. The multiple scatterings of the pair give rise to the unitarization of the corresponding cross section. The calculations are performed in the double logarithmic approximation (DLA), corresponding to the condition $\alpha_{s} \ll \gamma_{G} \ll 1$, where $\alpha_{s}$ and $\gamma_{G}$ are the QCD coupling constant and the gluon anomalous dimension, respectively. In this approximation, the transverse separation of the pair remains fixed allowing an eikonal description for the interaction gluon pair-nucleon through incoherent multiscatterings [10]. The cross section for the interaction can be expressed in terms of the nucleon gluon distribution $x G\left(x, \widetilde{Q}^{2}\right)$ and of the transverse separation $r$ of the gluon pair. The procedure for an initial state quarkantiquark pair is similar, up to proper color coefficients. Such a formulation has produced comprehensive phenomenological applications: the inclusive structure function $F_{2}$ [12], the longitudinal $F_{L}$ and charmed $F_{2}^{c \bar{c}}$ ones [13], the logarithmic slope $\partial F_{2} / \partial \log Q^{2}[14]$, and other related quantities have been calculated in the Breit system. The respective nuclear case has been investigated in Refs. [15] and the equivalence with other high density QCD approaches has been reported in Refs. [16]. Moreover, the asymptotic limit of the inclusive structure function and its logarithmic slope are estimated in [17]. Regarding the rest frame, Glauber-Mueller has been also used to estimate the saturation effects for DIS and diffractive dissociation [18] as well as being considered as an initial condition for a high energy evolution equation (for a review, see [7]). Therefore, the Glauber-Mueller (GM) approach gives a good framework for the unitarity effects (saturation) in the nucleon and nuclear sectors, providing the dynamics of the observables in a quantitative level.

In this work we make use of the parton saturation formalism to study the description of the observables driven by the gluonic content of the proton in the color dipole picture. The inclusive structure function $F_{2}$ is calculated properly, disregarding approximations commonly considered in previous calculations $[12,14,17]$. The structure functions $F_{L}$ and $F_{2}^{c \bar{c}}$ are presented for the first time using the Glauber-Mueller approach and the rest frame in comparison with the experimental data. The saturation effects are included in the effec- tive dipole cross section corresponding to the small size dipole contributions. The large dipole sizes are taken into account through an Ansatz for the nonperturbative region. Here, we choose to freeze the gluon distribution at large distances [18]. The dipole framework provides a clear identification in the transverse distance $r$ range where the perturbative and nonperturbative sectors (soft domain) contribute to the calculated quantities. Large dipoles correspond to the soft domain and the small ones are connected with the hard sector. As we will see, the photon wave functions play the role of a weight function selecting small dipole sizes, with a non-negligible contribution coming from the large dipole sizes. However, when considering the production of heavy quark pairs (charm and bottom), their masses largely diminish the soft contribution. These issues are addressed throughout this paper.

This work is organized as follows. In the next section we shortly review the deep inelastic scattering in the rest frame, introducing the main formulas for the further analysis. In Sec. III one addresses the effective dipole cross section considering saturation effects (unitarity corrections) encoded in the Glauber-Mueller approach, pointing out its main properties and discussing the large transverse separation contribution. Section IV is devoted to calculating the theoretical estimates for the HERA observables, focusing on those ones dominated by the gluon content. In Sec. V we draw our conclusions and comments.

\section{DEEP INELASTIC SCATTERING IN THE PROTON REST FRAME}

In the reference frame where the target (proton) has infinite momentum the usual description of the dynamics evolution is the following: the emitted partons from the target remain quasifree for enough time in such a way that the virtual probe (photon) detects them as real particles (asymptotic freedom). On the other hand, in the small $x$ region it is suitable to describe the evolution in a system where the target is at rest: in this situation the evolution is related to the partonic fluctuations of the probe and their interactions with the nucleon. The rest frame physical picture is advantageous since the lifetimes of the photon fluctuation and of the interaction process are well defined [19]. The simplest case is the quark-antiquark state (color dipole), which is the leading configuration for small transverse size systems. Its lifetime can be estimated by the uncertainty principle through the energy fluctuation associated with the emerging pair. The well known coherence length is expressed as $l_{c}=1 /\left(2 x m_{p}\right)$, where $x$ is the Bjorken variable and $m_{p}$ the proton mass. For instance, in deep inelastic at HERA kinematics reaching $x$ $\sim 10^{-5}$, the coherence length is about $10^{4} \mathrm{fm}$, which is a distance larger than the radius of any atomic nuclei. An immediate consequence of such a picture is the factorization between virtual photon wave functions and the interaction cross section on the corresponding amplitude in the impact parameter space representation. (See Fig. 1.)

A striking consequence of the formulation above is that the photoabsortion cross section can be derived from the expectation value of the interaction cross section for the mul- 


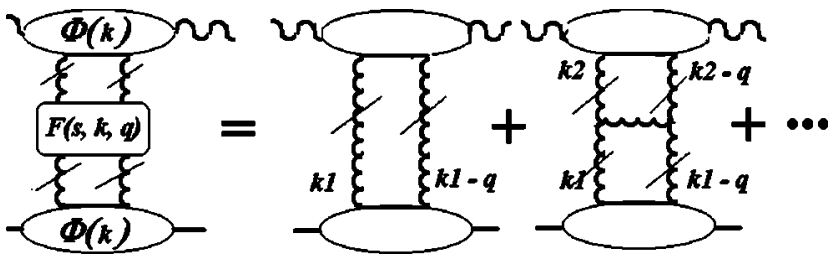

FIG. 1. Representation of the deep inelastic process, where the upper blob corresponds to the photon impact factor and the bottom one represents the proton impact factor. The corresponding two first orders in perturbative expansion (BFKL-like) are depicted.

tiparticle Fock states of the virtual photon weighted by the light-cone wave functions of these states [5]. The scattering matrix has an exact diagonalization in the $\gamma^{*}$ Fock states representation, where the partial-wave amplitudes subjected to the $s$-channel unitarization are identified properly. Then, the photoabsorption cross section can be cast into the quantum mechanical factorized form,

$$
\sigma_{T, L}^{\gamma^{*} p}\left(x, Q^{2}\right)=\int d^{2} \mathbf{r} \int_{0}^{1} d z\left|\Psi_{T, L}(z, \mathbf{r})\right|^{2} \sigma^{\text {dipole }}(x, z, \mathbf{r}) .
$$

The formulation above is valid even beyond perturbation theory, since it is determined from the space-time structure of the process. The $\Psi_{T, L}(z, \mathbf{r})$ are the photon wave functions (for transverse $T$ and longitudinal $L$ polarizations) describing the pair configuration, where $z$ and $1-z$ are the fraction of the photon's light-cone momentum carried by the quark and antiquark of the pair, respectively. The transverse separation of the pair is $\mathbf{r}$. The precise normalizations of the wave functions can be determined through the Fock expansion $\left|\gamma_{\text {phys }}\right\rangle$ $=\sqrt{Z_{3}}\left|\gamma_{\text {bare }}\right\rangle+\mathcal{N}_{q \bar{q}}|q \bar{q}\rangle$, where $Z_{3}$ is the $\gamma$ wave function renormalization constant, $\left|\gamma_{\text {bare }}\right\rangle$ denotes the bare state and $\mathcal{N}_{q \bar{q}}$ is the coefficient determining the probability of the $q \bar{q}$ pair fluctuation in the photon [20]. Considering completely normalized states, then $\mathcal{N}_{q \bar{q}}^{2}=1-Z_{3}$ and the normalization for transverse photons is obtained from $\int d z d^{2} \mathbf{r}\left|\Psi_{T, L}(z, \mathbf{r})\right|^{2}=\mathcal{N}_{q \bar{q}}^{2}$. The remaining normalizations, i.e., longitudinal component and cross section, are consequently fixed [20]. The explicit expressions are well known,

$$
\begin{aligned}
\left|\Psi_{T}(z, \mathbf{r})\right|^{2}= & \frac{6 \alpha_{\mathrm{em}}}{4 \pi^{2}} \sum_{i}^{n_{f}} e_{i}^{2}\left\{\left[z^{2}+(1-z)^{2}\right] \varepsilon^{2} K_{1}^{2}(\varepsilon r)\right. \\
& \left.+m_{q}^{2} K_{0}^{2}(\varepsilon r)\right\}
\end{aligned}
$$

and

$$
\left|\Psi_{L}(z, \mathbf{r})\right|^{2}=\frac{6 \alpha_{\mathrm{em}}}{4 \pi^{2}} \sum_{i}^{n_{f}} e_{i}^{2}\left\{4 Q^{2} z^{2}(1-z)^{2} K_{0}^{2}(\epsilon r)\right\}
$$

Clarifying the notation, the auxiliary variable $\varepsilon^{2}=z(1$ $-z) Q^{2}+m_{q}^{2}$, with $m_{q}$ the light quark mass, and $K_{0}$ and $K_{1}$ are the McDonald functions of rank zero and one, respectively.
The quantity $\sigma^{\text {dipole }}(x, z, \mathbf{r})$ is interpreted as the cross section of the scattering of the effective dipole with fixed transverse separation $\mathbf{r}$ [5]. It is directly dependent on the unintegrated gluon distribution,

$$
\sigma^{\text {dipole }}(x, z, \mathbf{r})=\frac{4 \pi \alpha_{s}}{3} \int \frac{d^{2} \mathbf{k}_{1}}{\mathbf{k}_{1}^{4}} \mathcal{F}\left(\frac{x}{z}, \mathbf{k}_{1}\right)\left(1-e^{i \mathbf{k}_{1} \cdot \mathbf{r}}\right) .
$$

The unintegrated gluon distribution, $\mathcal{F}\left(x / z, \mathbf{k}_{1}\right)$, vanishes at the gluon transverse momentum $\left|\mathbf{k}_{1}\right| \rightarrow 0$ [and similarly for the factor $\left.\left(1-e^{i \mathbf{k}_{1} \cdot \mathbf{r}}\right)\right]$ due to gauge invariance, and therefore the dipole cross section has to be infrared finite. The most important feature of the dipole cross section is its universal character, namely it depends only on the transverse separation $\mathbf{r}$ of the color dipole. The dependence on the external probe, i.e., the photon virtuality, is included in the wave functions.

The main technical difficulty in Eq. (4) is to model the unintegrated gluon distribution function in a suitable way, mainly in the small transverse momentum $\left(k_{T}\right)$ region (infrared sector). In particular, to obtain these distributions one should solve numerically an evolution equation, which makes the procedure cumbersome for practical use (instead, for a prompt parametrization see [21]). In general, this is avoided introducing an Ansatz for the effective dipole cross section and analyzing the process in the impact parameter space. The main feature of the current models in the literature is to interpolate the physical regions of small transverse separations (QCD-parton improved model picture) and the large ones (Regge-soft picture). Below, we quote two of them, which have connections with the work performed here.

The phenomenological saturation model of Golec-Biernat and Wüsthoff (GBW) gives a good description of DIS data [23]. The corresponding cross section interpolates between color transparency, i.e., $\sigma^{\text {dipole }} \sim r^{2}$ at small $r$, and the constant cross section $\sigma_{0}$ at large $r$ (confinement). Such a procedure ensures $Q^{2}$ saturation, while parton saturation at low $x$ is obtained with an eikonal-inspired shape for the dipole cross section,

$$
\begin{aligned}
\sigma^{\mathrm{GW}}\left(x, r^{2}\right) & =\sigma_{0}\left(1-e^{-r^{2} / R_{\mathrm{sat}}^{2}(x)}\right), \\
R_{\mathrm{sat}}^{2}(x) & =4 R_{0}^{2}(x)=\frac{4}{Q_{0}^{2}}\left(\frac{x}{x_{0}}\right)^{\lambda},
\end{aligned}
$$

where the parameters $\sigma_{0}=23.03 \mathrm{mb}, x_{0}=3.0410^{-4}$, and $\lambda=0.288$ are fitted to the HERA DIS inclusive data with $x$ $<10^{-2}$, whereas $Q_{0}^{2}=1 \mathrm{GeV}^{2}$ sets the dimension. The $x$-dependent saturation radius $R_{0}^{2}(x)$ scales the pair separation $r$ in the cross section and is associated with the mean separation between partons in the nucleon. This approach was used to describe diffractive dissociation in a parameterfree way, considering also the required $q \bar{q} G$ configuration. Some criticism of the GBW model, mainly concerning a better knowledge of the parton (gluon) distribution at low $r$ and its saturation at small $x$ are postponed to the next section. Most of them can also be found in Refs. $[3,18]$. 
Our work in this paper is closer to the McDermottFrankfurt-Guzey-Strikman (MFGS) one [24] where perturbative QCD relates the dipole cross section to the leading logarithmic gluon distribution function in the proton, at $\operatorname{LLA}\left(Q^{2}\right)$ accuracy,

$$
\sigma^{\mathrm{MFGS}}=\frac{\pi^{2} \alpha_{s}\left(\widetilde{Q}^{2}\right)}{3} r^{2} x G\left(x, \widetilde{Q}^{2}\right)
$$

where the identification $\widetilde{Q}^{2} \approx Q^{2}$ is allowed at the leading$\log$ arithmic level. A study of the dipole cross section is carried for all transverse separations $r$ in [24]. The small $r$ region is described by the usual gluon PDF's, evoluting with the scale $\widetilde{Q}^{2}=10 / r^{2}$, while for large separations ( $r_{\text {pion }}$ $\geqslant 0.65 \mathrm{fm}$ ) the dipole cross section is driven by the pionproton contribution with the typical soft energy behavior from the hadronic sector. Moreover, the taming of the parton density is implemented by hand starting at the named critical transverse separation $r_{\text {crit }}$, stated when the dipole cross section reaches one-half of its maximum value labeled by the pion-proton cross section. In connection with the present work, the Glauber-Mueller approach gives Eq. (6) at the Born level. Instead of using an ad hoc control of the gluon distribution, the Glauber-Mueller provides corrections required by unitarity in an eikonal expansion. For the large $r$ region, we choose to follow a similar procedure from the GBW model, namely saturating the dipole cross section ( $r$-independent constant value).

Having defined the notation and reviewed the main properties settled by the rest frame representation of the deep inelastic process, in the next section we address the unitarity corrections formalism contained in the Glauber-Mueller approach that we will consider in the calculation of $F_{2}, F_{L}$, and $F_{2}^{c \bar{c}}$.

\section{THE GLAUBER-MUELLER APPROACH}

The Glauber formalism concerns mainly interactions with a target nucleus, allowing us to calculate the amount of unitarity corrections to the nuclear cross section. However, this approach can be extended to take into account the evolution of the partonic densities (saturation) through the multiple scatterings. Below, we review shortly the main properties of the Glauber formalism in QCD, in the nuclear case as its application to the nucleon case. We indicate the original papers [10] for a complete presentation. It should be stressed that saturation effects mean unitarity corrections to the observables. Indeed, the asymptotic calculations have produced a unified $\ln (1 / x)$ pattern for the cross section and gluon function instead of an effectively saturated one [17].

Since the small $x$ limit is driven by the gluonic interactions, we consider a virtual probe $G^{*}$ with invariant mass $Q^{2}$ which decays into a gluon pair $G G$ having a transverse separation $\mathbf{r}$ and transverse momentum $\mathbf{k}$. Then, the pair interacts with the target through a gluonic ladder at fixed transverse separation. Following the discussion from Sec. II, the photoabsorption cross section for the probe particle in terms of $x$ and virtuality $Q^{2}$ in the nuclear case is

$$
\begin{aligned}
\sigma_{\text {tot }}^{G^{*} A}\left(x, Q^{2}\right)= & \int d^{2} \mathbf{r} \int_{0}^{1} d z\left|\Psi_{G G}\left(z, \mathbf{r}, Q^{2}\right)\right|^{2} \\
& \times \sigma^{G G-\text { nucleus }}(x, z, \mathbf{r}),
\end{aligned}
$$

where the variables have the same identification as in the $q \bar{q}$ pair discussion. The quantity $\Psi_{G G}$ is the light-cone wave function for the gluon pair. The Glauber multiscattering theory employs the phase shift method to describe processes at high energy for an incident particle undergoing into successive scatterings. When $x$ is small, the coherence length $l_{c}$ is bigger than the mean radius $R_{A}$ for all nuclei and the interaction of the initial parton stands through the entire nuclear path, providing coherent interactions with all target partons along the distance $l_{c}$. These scatterings are coherent in an interaction of a hadronic fluctuaction, i.e., quark or gluon pairs, with the nucleus. There are interference effects among them, generating a reduction in the nuclear cross section, $\sigma^{\text {nucleus }}<A \sigma^{\text {nucleon }}$. Otherwise, when $l_{c} \leqslant R_{A}$, full incoherent scatterings occur leading to the expectation that the nuclear cross section equals $A \sigma^{\text {nucleon }}$. The well-known Glauber formula for the total cross section of a hadronic state with the nucleus is

$$
\sigma_{\text {tot }}^{\text {nucleus }}=2 \int d^{2} \mathbf{b}\left(1-e^{-(1 / 2) \sigma_{\text {nucleon }} \mathrm{S}_{\mathrm{A}}(\mathbf{b})}\right),
$$

where $S_{A}(\mathbf{b})$ is a profile function containing the dependence on the impact parameter $\mathbf{b}$, which is the conjugate variable to the momentum transfer $t$. It is related to the nucleon distribution inside the nucleus and encodes the information about the angular distribution of the scattering. We discuss its particular shape later on. Equation (7) is quite general and allows us to describe the hadronic fluctuations of the photon or any virtual probe, as seen in Sec. II.

Considering the scattering amplitude dependent on the usual Mandelstan variables $s$ and $t$, now written in the impact parameter representation $\mathbf{b}$,

$$
a(s, \mathbf{b}) \equiv \frac{1}{2 \pi} \int d^{2} \mathbf{q} e^{-i \mathbf{q} \cdot \mathbf{b}} \mathcal{A}\left(s, t=-q^{2}\right),
$$

the corresponding total and elastic cross sections (from the optical theorem) are rewritten in the impact parameter representation (b) as

$$
\begin{aligned}
\sigma_{\text {tot }} & =4 \pi \operatorname{Im} \mathcal{A}(s, 0)=2 \int d^{2} \mathbf{b} \operatorname{Im} a(s, \mathbf{b}), \\
\sigma_{e l} & =\int d^{2} \mathbf{b}|a(s, \mathbf{b})|^{2} .
\end{aligned}
$$

A very important property when treating the scattering in the impact parameter space is the simple definition for the unitarity constraint [10]. At fixed $\mathbf{b}$, the constraint can be expressed in the following way:

$$
\begin{gathered}
\sigma_{t o t}=\sigma_{e l}+\sigma_{\text {inel }}, \\
2 \operatorname{Im} a(s, \mathbf{b})=|a(s, \mathbf{b})|^{2}+C_{i n}(s, \mathbf{b}),
\end{gathered}
$$


with $C_{i n}(s, \mathbf{b})$ denoting the sum of contributions from all the inelastic channels. The constraint above has a simple solution. If the real part of the scattering amplitude vanishes at the high energy limit, corresponding to small $x$ values, the solution is

$$
\begin{aligned}
a(s, \mathbf{b}) & =i\left[1-e^{-(1 / 2) \Omega(\mathrm{s}, \mathbf{b})}\right], \\
\sigma_{t o t} & =2 \int d^{2} \mathbf{b}\left[1-e^{-(1 / 2) \Omega(s, \mathbf{b})}\right],
\end{aligned}
$$

where the opacity $\Omega$ is an arbitrary real function and it should be determined by a detailed model for the interaction. The opacity function has a simple physical interpretation, namely $e^{-\Omega}$ corresponds to the probability that no inelastic scatterings with the target occur. To provide the connection with the Glauber formalism, the opacity function can be written in the factorized form $\Omega(s, \mathbf{b})=\Omega(s) S(\mathbf{b})$, considering $S(\mathbf{b})$ normalized as $\int d^{2} \mathbf{b} S(\mathbf{b})=1$ (for a detailed discussion, see i.e., [25]).

From Eqs. (15) and (8), we identify the opacity $\Omega(s$ $\left.\approx Q^{2} / x ; \mathbf{r}\right)=\sigma^{\text {nucleon }}(x, \mathbf{r})$. Moreover, it has been found that the same formalism for multiple scatterings can be applied to the nucleon case. To proceed, we should determine the $G G$ cross section. The gluon pair cross section is equivalent to the quark pair one, up to a color factor $\left(\sigma^{G G}=\frac{9}{4} \sigma^{q \bar{q}}\right)$. The ( $q \bar{q}$ pair) dipole-proton cross section is well known $[10,25]$, calculated starting from Eq. (4), and in the double logarithmic approximation (DLA) has the following form:

$$
\sigma_{\text {nucleon }}^{q \bar{q}}(x, r)=\frac{\pi^{2} \alpha_{s}\left(\widetilde{Q}^{2}\right)}{3} r^{2} x G\left(x, \widetilde{Q}^{2}\right)
$$

with the $r$-dependent scale $\widetilde{Q}^{2}=r_{0}^{2} / r^{2}$. Considering Eq. (16) one can connect directly the dipole picture with the usual parton distributions (gluon), since they are solutions of the DGLAP equations. In our case, we follow the calculations in Refs. $[10,25]$ and consider the effective scale $\widetilde{Q}^{2}=4 / r^{2}$. Such a value differs from [24], where it is $r_{0}^{2}=10$, which is obtained by an averaging procedure on the transverse size integral of $F_{L}$. However, in further studies in vector mesons it was found that $r_{0}^{2}$ ranges from $4-15$, and $F_{2}$ and $F_{L}$ are not sensitive to those variations. Thus, these values are consistent in leading logarithmic $Q^{2}$ approximation.

From the above expression, one obtains a dipole cross section satisfying the unitarity constraint and a framework to study the unitarity effects (saturation) in the gluon DGLAP distribution function. Hence, hereafter we use the GlauberMueller dipole cross section given by

$$
\sigma_{\text {dipole }}^{G M}=2 \int d^{2} \mathbf{b}\left(1-e^{-(1 / 2) \sigma_{\text {nucleon }}^{q \bar{q}}(\mathbf{x}, \mathbf{r}) \mathrm{S}(\mathbf{b})}\right) .
$$

Here, some comments are in order. The Glauber-Mueller approach is valid in the small $x$ region, and the gluon emission is described in the double logarithmic approximation of perturbative QCD. The interaction of the quark pair with the nucleon (proton) occurs through ladder diagrams exchange, satisfying the DGLAP equations in the DLA limit. The high energy limit allows us to treat successive scatterings as independent collisions, meaning the process described by the eikonal picture of a relativistic particle crossing the nucleus. Moreover, as a consequence of no correlation among nucleons inside the nucleus (in the nuclear case), there are no correlations among partons from different partonic cascades, stressing that only the fastest parton interacts with the target. The corrections coming from the slowest partons in the cascade (emitted by the pair) lead to the AGL nonlinear evolution equation [10], and they have been considered recently to describe diffractive DIS in Ref. [18]. Regarding criticisms to the Glauber-Mueller approach, we indicate the recent paper [26] for a complete discussion about the eikonal-like models, concerning their advantages and limitations as well as pointing out the improvements to be taken into account to introduce the proper corrections.

Now, we proceed to calculate numerical estimates of the dipole cross section using the Glauber-Mueller approach through Eqs. (16), (17). Then we are calculating saturation effects in the color dipole picture. Firstly, we need to discuss the profile function $S(b)$. This function contains information about the angular distribution in the scattering, namely the $t$ dependence (quark pair-ladder and proton-ladder couplings). Both of them can be approximated by an exponential parametrization, leading to a simple Gaussian shape in the impact parameter space, $S(b)=\left(A / \pi R_{A}^{2}\right) e^{\left(-b^{2} / R_{A}^{2}\right)}$, where $A$ is the atomic number and $R_{A}$ is the target radius. We will keep this notation although we are only concerned with the nucleon case. The $R_{A}^{2}$ value should be determined from data, ranging between 5-10 $\mathrm{GeV}^{-2}$ for the proton case (see discussions in Refs. $[7,18]$ ). For nuclear reactions, a more suitable shape for the profile should be taken into account, since the Gaussian approximation is no longer appropriate to describe the nuclear profile for large $A$. Here, we have used the value $\left(R_{A}^{2}=5 \mathrm{GeV}^{-2}\right)$ obtained from a good description of both the inclusive structure function and its derivative [14]. Such a value corresponds to significative unitarity corrections to the standard DGLAP input even in the current HERA kinematics.

Now, we discuss in a detailed way the main characteristics emerging from the dipole cross section Eq. (17). In order to do this, in Fig. 2 one shows the Glauber-Mueller dipole cross section as a function of dipole transverse size $r$ at fixed momentum fraction $x$. For a better illustration of the expected partonic saturation effects, we run the Bjorken $x$ down to a quite small value $x=10^{-7}$ (THERA region). Hereafter, we are using the GRV gluon distribution at leading order [27] in the input Eq. (16), whose choice we justify below. The solid lines correspond to the dipole cross section calculation, Eq. (17), whereas the dashed lines are the GBW model [23] presented for comparison. The general shape in terms of the dipole size comes from the balancing between the color transparency $\sigma_{d i p} \sim r^{2}$ behavior and the gluon distribution.

Here some comments about the large transverse separation are in order: although perturbative QCD provides reliable results at small distances (small dipole sizes), the non- 


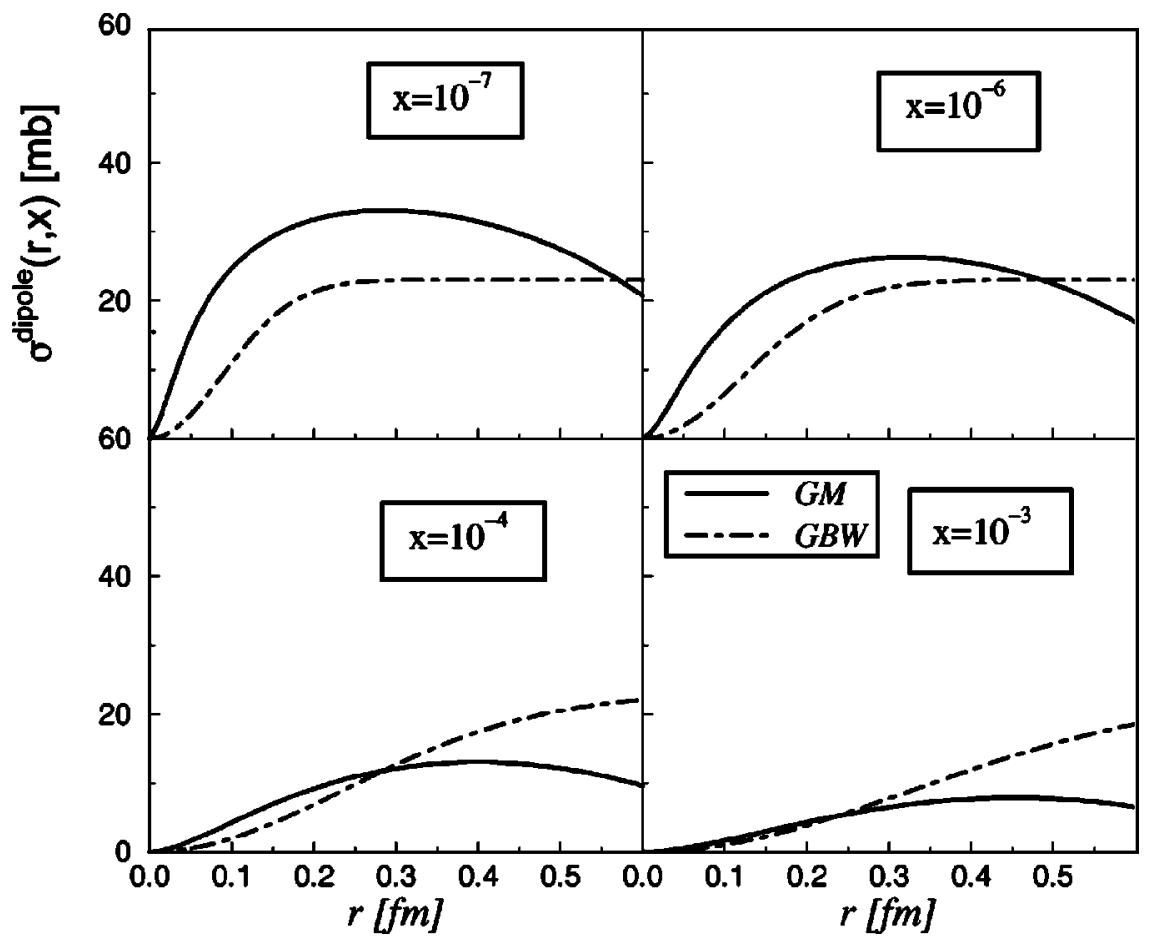

FIG. 2. The dipole cross section as a function of $r$ at fixed $x$. The Glauber-Mueller corresponds to the solid line and the GBW model results (dashed curves) are also included.

perturbative sector is still not completely understood. The usual PDF's are evoluted from a perturbative initial scale $Q_{0}^{2} \approx 1 \mathrm{GeV}^{2}$, and one has little information about the behavior at $Q^{2} \leqslant Q_{0}^{2}$. In general one makes use of Regge phenomenology to estimate those contributions (see, for instance [24]). Thus, extrapolating to lower virtuality regions (large dipole sizes) one needs a parametrization regarding the nonperturbative sector.

This is the main justification of the use the Glück-ReyaVogt 1994 (GRV94) parametrization [27] in our calculations. Bearing in mind that $Q^{2}=4 / r^{2}$, its evolution initial scale is $Q_{0}^{2}=0.4 \mathrm{GeV}^{2}$, allowing us to scan dipole sizes up to $r_{\text {cut }}$ $=\left(2 / Q_{0}\right) \mathrm{GeV}^{-1}(=0.62 \mathrm{fm})$. For the most recent parametrizations, where $Q_{0}^{2} \sim 1 \mathrm{GeV}^{2}\left(r_{\text {cut }} \approx 0.4 \mathrm{fm}\right)$ the amount of nonperturbative contribution in the calculations should increase. An additional advantage is that GRV94 does not include nonlinear effects to the DGLAP evolution since the parametrization was obtained from rather large $x$ values. This feature ensures that the parametrization does not include unitarity corrections (perturbative shadowing effects) in the initial scale.

Now, we should introduce an Ansatz for the large transverse separation region. A more phenomenological way is to match the PQCD dipole cross section with the typical hadronic one $\sigma_{\pi N}$ at $r_{\text {cut }}$ as performed in [24]. However, due to the significant growth of the PQCD dipole cross section at high energies, we choose an alternative Ansatz: the gluon distribution is frozen at scale $r_{\text {cut }}$, namely $x G\left(x, \widetilde{Q}_{\text {cut }}^{2}\right)$. Then, for the large distance contribution $r \leqslant r_{\text {cut }}$ the gluon distribution reads as

$$
x G\left(x, Q^{2} \leqslant Q_{0}^{2}\right)=\frac{Q^{2}}{Q_{0}^{2}} x G\left(x, Q^{2}=Q_{0}^{2}\right),
$$

leading to the correct behavior $x G \sim Q^{2}$ as $Q^{2} \rightarrow 0$. In a more sophisticated case, one can substitute the frozen scale $\widetilde{Q}_{\text {cut }}^{2}$ by the saturation scale $Q_{s}^{2}(x)$ to take into account a realistic value of the gluon anomalous dimension in all kinematic region (see correlated issues in [18]).

Recently, the phenomenological model of Ref. [23] has produced a good description of HERA data in both inclusive and diffractive processes. It is constructed interplaying the color transparency behavior $\sigma_{d i p} \sim r_{\perp}^{2}$ at small dipole sizes and a flat (saturated) behavior at large dipole sizes $\sigma_{d i p}$ $\sim \sigma_{0}$ (confinement). The expression has the eikonal-like form

$$
\sigma_{q q}(x, r)=\sigma_{0}\left[1-\exp \left(\frac{r^{2} Q_{0}^{2}}{4\left(x / x_{0}\right)^{\lambda}}\right)\right],
$$

where $Q_{0}^{2}=1 \mathrm{GeV}^{2}$ and the three fitted parameters are $\sigma_{0}$ $=23.03 \mathrm{mb}, x_{0}=3.04 \times 10^{-4}$, and $\lambda=0.288$ and the notation for the saturation radius $R_{0}(x)=\left(x / x_{0}\right)^{\lambda / 2}$. The GBW total cross section lies below the typical hadronic cross section: for instance in the pion-proton case, convoluting the pion wave function squared with the GBW dipole cross section we would have a constant cross section at high energies $\sigma_{\text {tot }}^{G B W} \leqslant \sigma_{\text {tot }}^{\pi N}$. Despite describing data in good agreement, GBW has some details that deserve some discussions: the approach does not present a dynamical hypothesis for the saturation phenomena and does not match DGLAP evolution. In GBW, saturation is characterized by the $x$-dependent saturation radius $Q_{s}^{2}(x)=1 / R_{0}^{2}(x)$ instead of the scale coming from Glauber-Mueller, $\kappa_{G}\left(x, Q_{s}^{2}\right)=1$, which can be easily extended for the nuclear case [10].

In Fig. 2 one shows the Glauber-Mueller dipole cross section and the GBW model [23]. We choose to compare them 
due to the fact that GBW is actually a particular case of the Glauber-Mueller approach, considering an oversimplified profile function. We should show below that this fact allows us to construct an extended saturation model with DGLAP evolution [28] (the BGK model). In the lower plots, where $x=10^{-3}$ and $x=10^{-4}$, the GM cross section underestimates the GBW one. However, as $x$ decreases the gluon distribution in the proton rises producing a bigger dipole cross section. This feature is clear in the upper plots, for smaller $x=10^{-6}$ and $x=10^{-7}$, where GM overestimates GBW by a significant factor mostly at intermediate $r$ values.

Finally, we show the connection between the GlauberMueller approach with the saturation model with DGLAP evolution [28], observing that it is timely since the latter is quite efficient in describing data and its qualitative success corroborates quantitative QCD studies. Considering the particular case of central collisions, namely scattering at impact parameter $b=0 \quad\left(S=A / \pi R_{A}^{2}\right)$, the Glauber-Mueller approach produces

$$
\sigma_{\text {dipole }}^{G M}(x, \mathbf{r}, b=0)=2 \int d^{2} \mathbf{b}\left(1-e^{-(1 / 2) \sigma_{\text {nucleon }}^{q \bar{q}}(x, \mathbf{r})\left(1 / \pi R_{\mathrm{A}}^{2}\right) .}\right.
$$

The integration over $b$ can be promptly carried out, and introducing the notation for the proper normalization for the dipole cross section, $\sigma_{0} \equiv 2 \pi \int_{0}^{R_{A}^{2}} d b^{2}=2 \pi R_{A}^{2}$, Eq. (20) recovers the simple expression for the saturation model DGLAP evoluted [28]:

$$
\sigma_{\text {dipole }}^{B G K}(x, \mathbf{r})=\sigma_{0}\left[1-\exp \left(-\frac{\pi^{2} \mathbf{r}^{2} \alpha_{s}\left(\mu^{2}\right) x G\left(x, \mu^{2}\right)}{3 \sigma_{0}}\right)\right] .
$$

For a phenomenological analysis, the parameter $\sigma_{0}$ and the scale $\widetilde{Q}^{2}$ are determined from data in [28]. In our case, it assumes the well defined value $\sigma_{0}=12.22 \mathrm{mb}$, using $R_{A}^{2}=5 \mathrm{GeV}^{-2}$. For a larger radius, for instance $R_{A}^{2}$ $=10 \mathrm{GeV}^{-2}$, one obtains a value $\sigma_{0}=24 \mathrm{mb}$, closer to the GBW one. Here, the virtuality scale is $\widetilde{Q}^{2}=4 / r^{2}$, whereas BGK choose the parametric form $\widetilde{Q}^{2} \equiv \mu^{2}=C / r^{2}+\mu_{0}^{2}$ and a two-parameter initial condition for the gluon distribution function.

\section{UNITARITY EFFECTS IN ep COLLISIONS}

This section is devoted to the study and estimate of the gluon-driven observables measured at the HERA kinematical domain in the rest frame. The first one is the inclusive structure function $F_{2}\left(x, Q^{2}\right)$, the main quantity testing the small $x$ physics. The unitarity corrections are well established for this observable considering the Glauber-Mueller approach [12] as well as its high energy asymptotics [17], namely the black disk limit. We review these issues considering the dipole picture (rest frame), using a more complete analysis similar to [23,24], but mostly, we discuss in detail the role played by the nonperturbative physics needed to describe the structure function, and where in the transverse separation $r$ range it starts to be important.

The longitudinal structure function $F_{L}\left(x, Q^{2}\right)$ is also addressed, verifying the frame invariance in comparison with previous laboratory frame calculations [13]. The longitudinal wave function strongly suppresses large $r$ contributions, thus selecting smaller nonperturbative contribution in comparison with the $F_{2}$ case. Moreover, $F_{L}$ is one of the main observables scanning possible higher twist corrections in the standard operator product expansion (OPE) [8]. Therefore, a reasonable description of this quantity suggests that the Glauber-Mueller formalism (or similar eikonal-like approaches) take into account the most important contributions to the complete higher-twist corrections at current kinematical regimes.

The structure function $F_{2}^{c \bar{c}}\left(x, Q^{2}\right)$ gives the charm quark content on the proton and is directly driven by the gluon distribution. Therefore it is a powerful observable to scan saturation effects in the small $x$ region. However, the current experimental status requires more dedicated measurements and better statistics. We verify a consistent description in the rest frame corroborating the similar analysis in the dipole models [22,29] and in those models considering unitarity corrections in the laboratory frame [13].

\section{A. The inclusive structure function $F_{2}\left(x, Q^{2}\right)$}

Now we perform estimates for the inclusive structure function in the rest frame considering the Glauber-Mueller dipole cross section. The expression for $F_{2}$, with the explicit integration limits on photon momentum fraction $z$ and transverse separation $r$, is

$$
\begin{aligned}
F_{2}\left(x, Q^{2}\right)= & \frac{Q^{2}}{4 \pi^{2} \alpha_{\text {em }}} \int_{0}^{\infty} d^{2} \mathbf{r} \int_{0}^{1} d z \\
& \times\left[\left|\Psi_{T}(z, \mathbf{r})\right|^{2}+\left|\Psi_{L}(z, \mathbf{r})\right|^{2}\right] \\
& \times \sigma_{\text {dipole }}^{G M}\left(x, \mathbf{r}^{2}\right) .
\end{aligned}
$$

The notation has been introduced in the preceding sections. In Fig. 3 one shows the estimates for the structure function for representative virtualities $Q^{2}$ from the latest H1 Collaboration measurements [30]. The longitudinal and transverse contributions are shown separately, the longitudinal one being subdominant as is well known. An effective light quark mass ( $u, d, s$ quarks) was taken, with the value $m_{q}=300 \mathrm{MeV}$, and the target radius is considered $R_{A}^{2}$ $=5 \mathrm{GeV}^{-2}$, in agreement with Ref. [14]. It should be stressed that this value leads to larger saturation corrections rather than using the radius ranging over $R_{A}^{2} \sim 8$ $-15 \mathrm{GeV}^{-2}$. The soft contribution comes from the freezing of the gluon distribution at large transverse separation as discussed in the preceding section. The gluon distribution considered is GRV94 at leading order [27], $x G^{\mathrm{GRV}}\left(x, 4 / r^{2}\right)$, whose choice has been justified in the preceding section.

From the plots we verify a good agreement in the normalization; however, the slope seems quite steep. This fact is due to the modeling for the soft contribution and it suggests that 

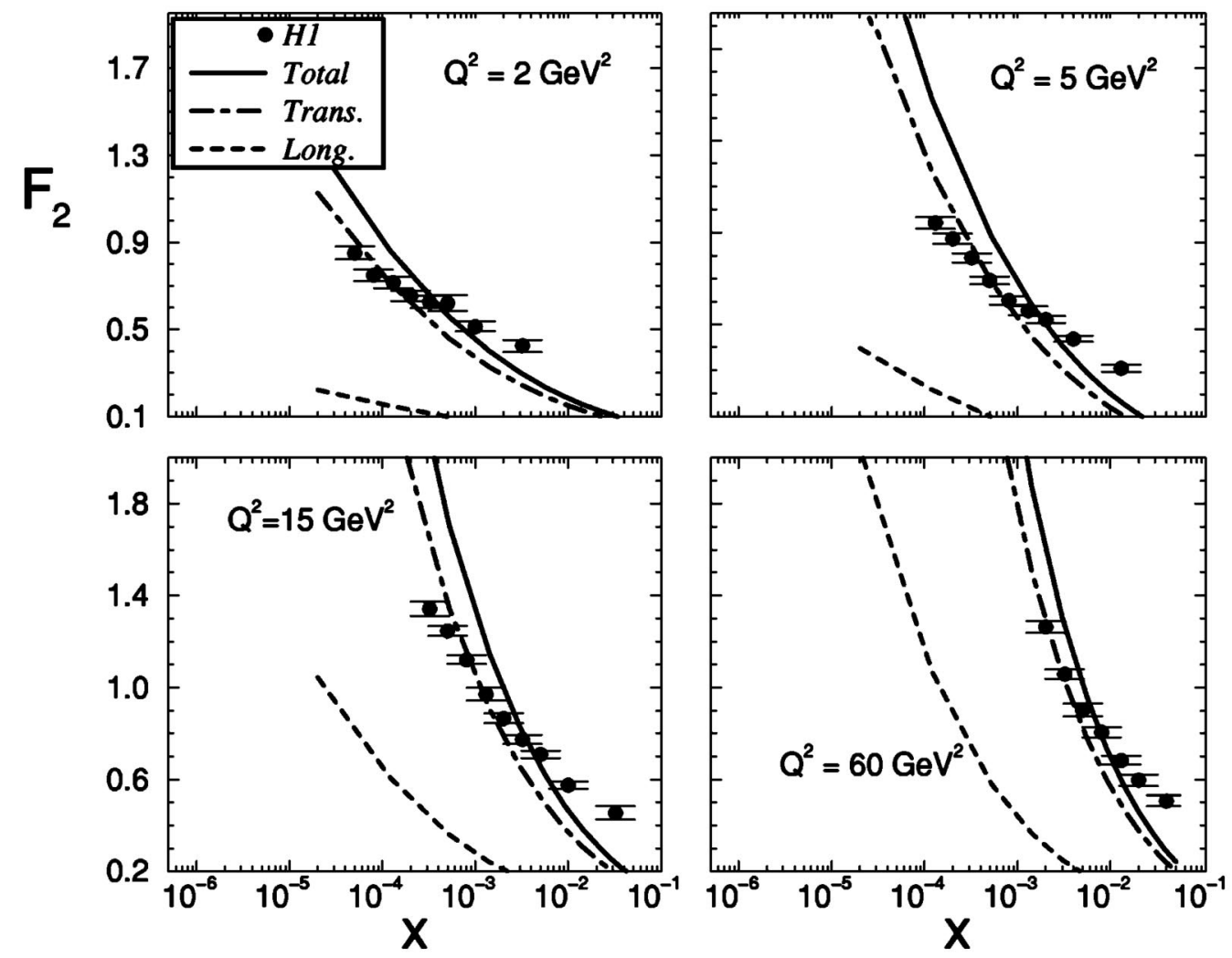

FIG. 3. The Glauber-Mueller result for the $F_{2}\left(x, Q^{2}\right)$ structure function. Shown are the transverse contribution (dot-dashed), the longitudinal contribution-(dashed), and the total-contribution (solid line). One considers light quarks, target radius $R_{A}^{2}=5 \mathrm{GeV}^{-2}$, and frozen gluon distribution at large $r>r_{\text {cut }}$. The input gluon distribution is GRV94LO [27] and data from H1 Collaboration [30].

a more suitable nonperturbative input should be taken. Indeed, in Ref. [24] such a question is addressed, claiming that the correct input is the pion-proton cross section parametrized through the Donnachie-Landshoff Pomeron. It is found that the large transverse separations give a larger contribution at low $Q^{2}$, whereas it vanishes concerning higher virtualities.

To clarify this issue, we can calculate the integrand $H_{q \bar{q}}\left(r, x, Q^{2}\right)$ used in the $r$ integration for the cross section $\sigma_{T}=\int_{0}^{\infty} d r H_{q \bar{q} T}\left(r, x, Q^{2}\right)$, which should have significant nonperturbative content. We plot in Fig. 4 this quantity at low virtuality $Q^{2}=2 \mathrm{GeV}^{2}$ and at a higher one $Q^{2}=30 \mathrm{GeV}^{2}$ for the momentum fraction with the range $10^{-4}<x<10^{-2}$, verifying that the main contribution comes from an asymmetric peak at $r \approx 0.15 \mathrm{fm}$ for $Q^{2}=2 \mathrm{GeV}^{2}$, while it is shifted to $r \approx 0.07$ at $Q^{2}=30 \mathrm{GeV}^{2}$. In our calculation, the perturbative contribution holds up to $r_{\text {cut }}=0.62 \mathrm{fm}$, therefore the region $r>r_{\text {cut }}$ gives a nonmarginal contribution to the cross section at low virtualities. Indeed, one has found that it reaches about $10 \%$ at $Q^{2}=2 \mathrm{GeV}^{2}$ and that when the virtuality increases the contribution gradually vanishes. In fact, using the most recent PDF's this situation is more criti-
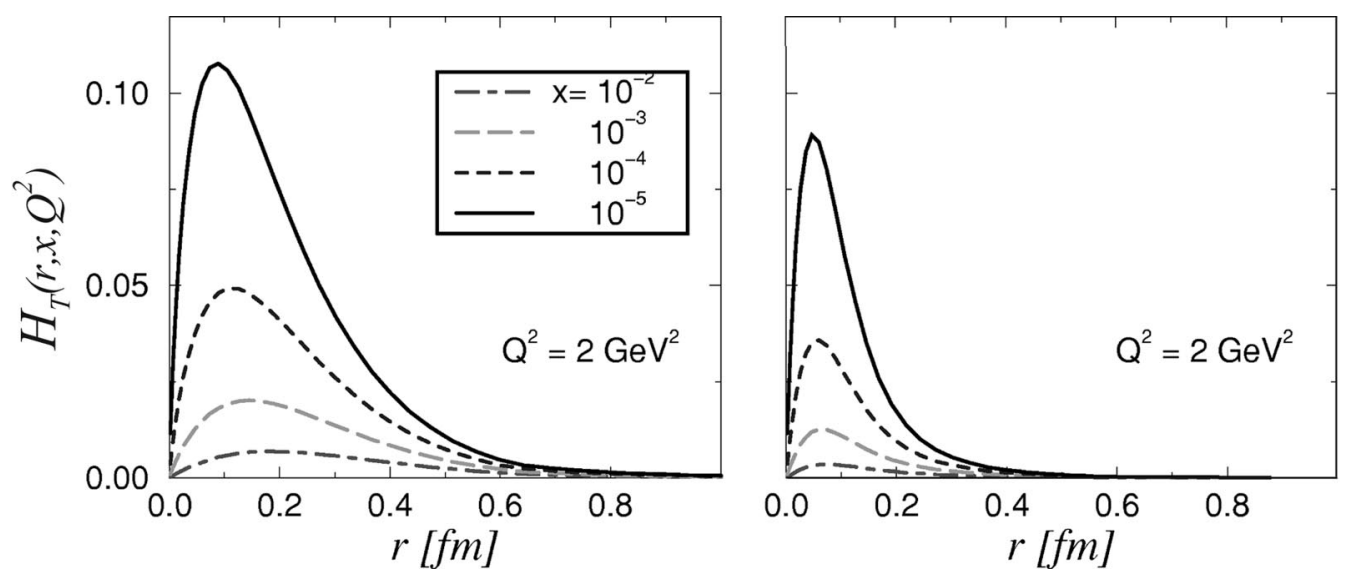

FIG. 4. The integrand $H_{q \bar{q} T}\left(r, x, Q^{2}\right)$ as a function of $r$ for $Q^{2}=2$ and $Q^{2}=30 \mathrm{GeV}^{2}$ at fixed $10^{-5}<x<10^{-2}$. 

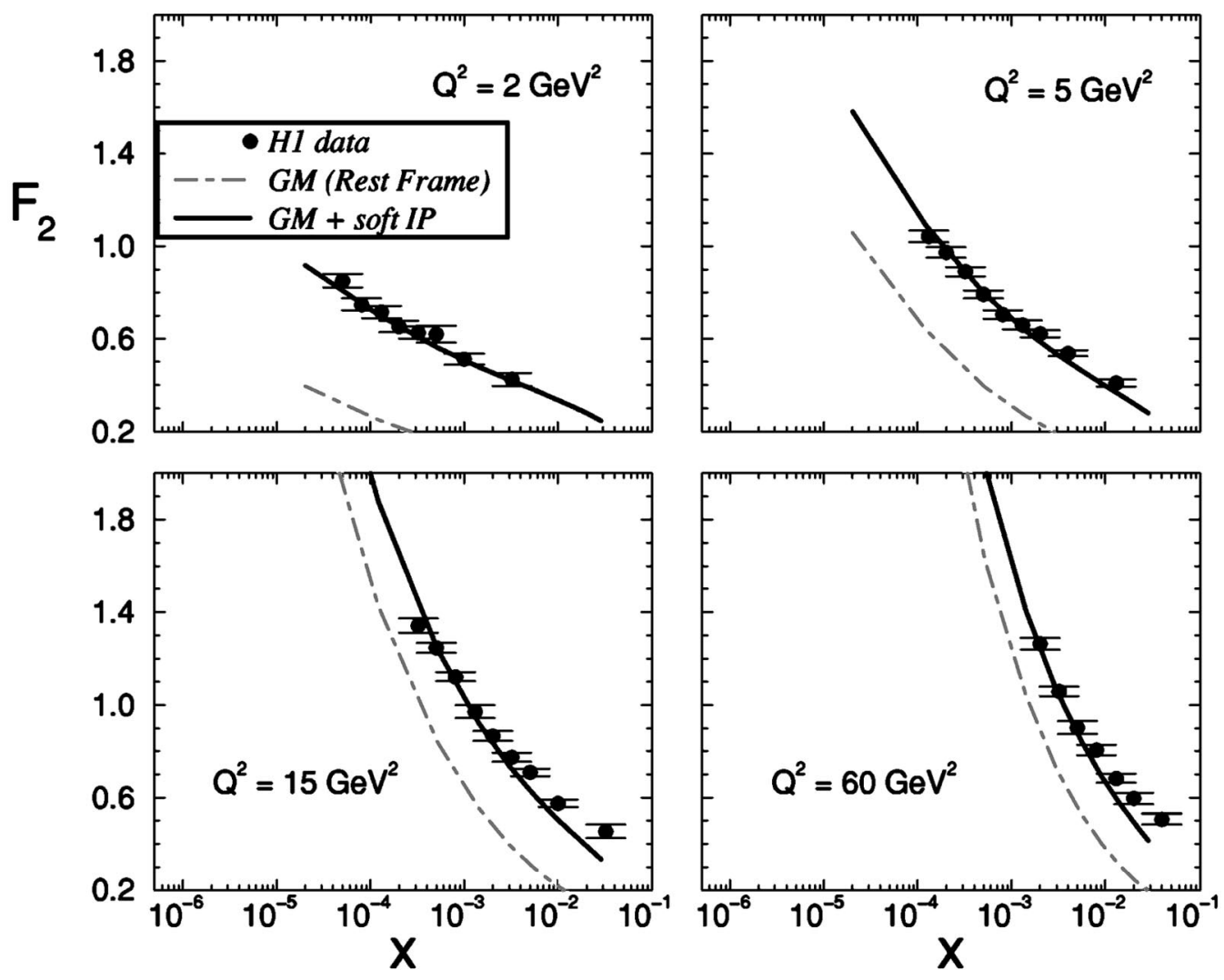

FIG. 5. The Glauber-Mueller prediction for the $F_{2}$ structure function in the rest frame. For the sake of comparison with the results in [12], one uses the quark sector $\left(R_{A}^{2}=5 \mathrm{GeV}^{-2}, m_{q}=0\right)$ and only the transverse wave function. Radius integration $1 / Q^{2}<r^{2}<1 / Q_{0}^{2}$ and soft Pomeron added [parametrizing the large pair separation $\left.-F_{2}^{\text {soft }}=\mathcal{C}_{\text {soft }} x^{-0.08}(1-x)^{10}\right][12]$.

cal since $r_{\text {cut }}$ is smaller $\left(Q_{0}^{2} \sim 1-2 \mathrm{GeV}^{2}\right)$. This suggests that the photon piece $\left|\Psi_{T}(z, r)\right|^{2}$ multiplying the dipole cross section enhances the $r$ integrand to smaller $r$ at high $Q^{2}$, corroborating a similar conclusion already found in Ref. [24].

To clarify the role played by the soft nonperturbative contribution to the inclusive structure function and to verify the frame invariance of the approach, in Fig. 5 we plot separately the perturbative contribution and parametrize the soft contribution introducing the nonperturbative structure function $F_{2}^{\text {soft }}=\mathcal{C}^{\text {soft }} x^{-0.08}(1-x)^{10}[12]$, which is added to the perturbative one. The soft piece normalization is $\mathcal{C}^{\text {soft }}=0.22$. Such a procedure is done in order to compare explicitly the results found in Ref. [12]. Accordingly, we have used just shadowing corrections for the quark sector, taking into account only the transverse photon wave function and zero quark mass. The integration on the transverse separation is taken for $1 / Q^{2} \leqslant r^{2} \leqslant 1 / Q_{0}^{2}$, with $Q_{0}^{2}=0.4 \mathrm{GeV}^{2}$ for the leading order GRV94 gluon distribution. This leads to a residual contribution absorbed in the the soft piece coming from transverse separations $r^{2}<1 / Q^{2}$. We considered the target radius being $R_{A}^{2}=5 \mathrm{GeV}^{2}$ (supported by [14]), which produces a correction more important than the value $R_{A}^{2}=10 \mathrm{GeV}^{2}$. It is again verified that the soft contribution is important at small virtualities and its importance decreases as it gets larger. In the plots, the dot-dashed lines represent only the perturbative calculations using the particular approximations indicated above, and the solid lines represent the results when we add the soft term.

Concluding, we have a theoretical estimate, i.e., no fitting procedure, of the inclusive structure function $F_{2}\left(x, Q^{2}\right)$ through the Glauber-Mueller approach for the dipole cross section, detecting a non-negligible importance of a suitable input for the large dipole size region.

\section{B. The longitudinal structure function $F_{L}\left(x, Q^{2}\right)$}

As we saw in Sec. II, the inclusive structure function can be expressed in terms of the cross sections $\sigma_{T}$ and $\sigma_{L}$ for the absortion of virtual photons transversally and longitudinally polarized, $F_{2}\left(x, Q^{2}\right)=\left(Q^{2} / 4 \pi \alpha_{\mathrm{em}}\right)\left(\sigma_{T}+\sigma_{L}\right)$. At small $x$, the longitudinal structure function is

$$
F_{L}\left(x, Q^{2}\right)=\frac{Q^{2}}{4 \pi \alpha_{\mathrm{em}}} \sigma_{L}\left(x, Q^{2}\right) .
$$

From QED, the longitudinal photons have zero helicity ( $h$ $=0)$ and therefore they have a virtual character. In the naive parton model, the helicity conservation for the electromagnetic vertex implies the Callan-Gross relation $F_{2}=2 x F_{1}$ and consequently a vanishing value for the longitudinal structure function $F_{L} \equiv F_{2}-2 x F_{1}$, considering the scattering photon quarks (spin 1/2). From QCD theory, this quantity has a nonzero value due to the gluon radiation, as is encoded in the Altarelli-Martinelli expression (see the discussion in [13]) 


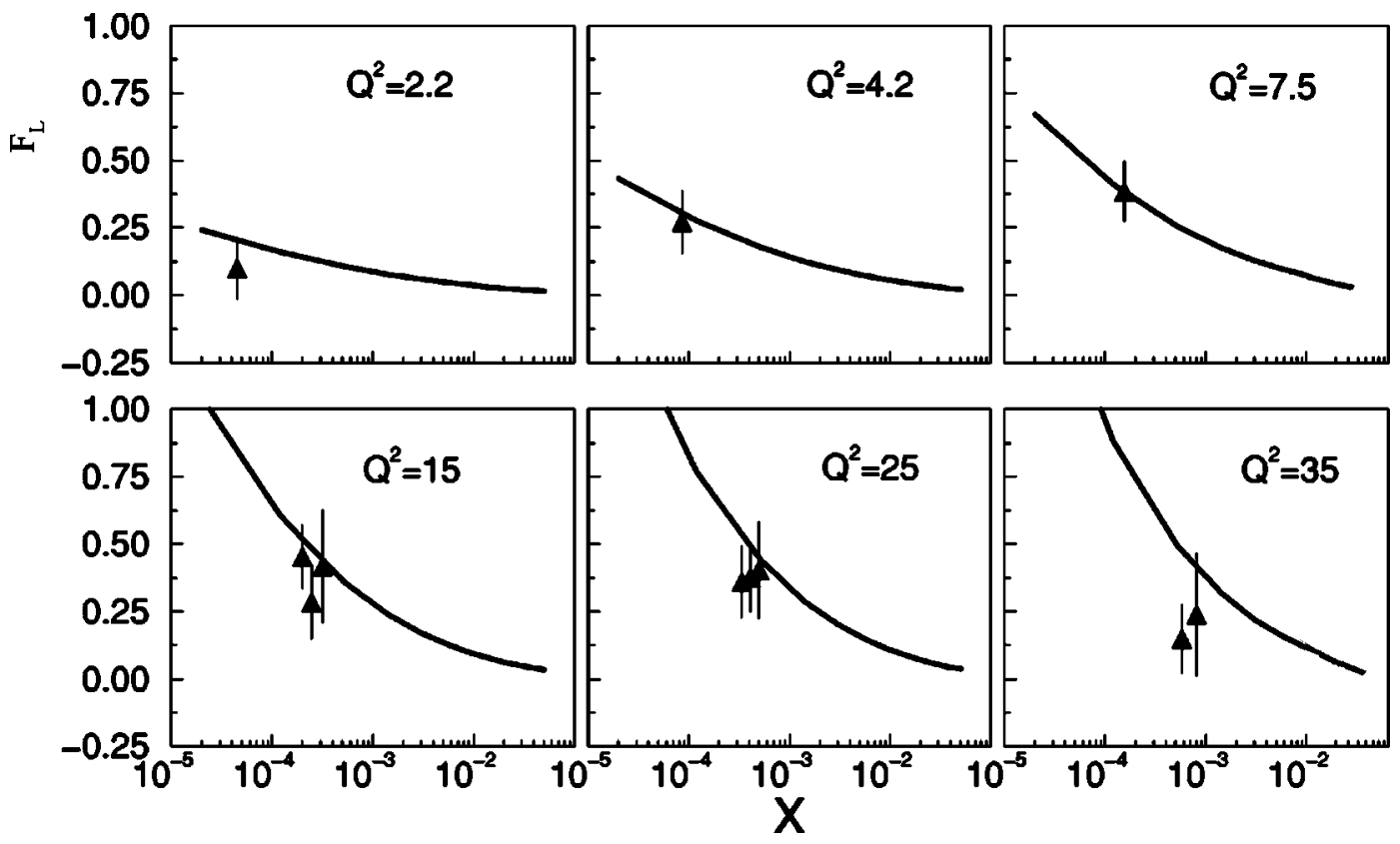

FIG. 6. The Glauber-Mueller estimates for the $F_{L}$ structure function. One uses light quarks $\left(m_{q}=300 \mathrm{MeV}\right)$, target size $R_{A}^{2}$ $=5 \mathrm{GeV}^{-2}$, and frozen gluon distribution at large $r$. Data are from the H1 Collaboration.

$$
\begin{aligned}
F_{L}\left(x, Q^{2}\right)= & \frac{\alpha_{s}\left(Q^{2}\right)}{2 \pi} x^{2} \int_{x}^{1} \frac{d y}{y^{3}}\left[\frac{8}{3} F_{2}\left(x, Q^{2}\right)\right. \\
& \left.+\frac{40}{9} y G\left(y, Q^{2}\right)\left(1-\frac{x}{y}\right)\right]
\end{aligned}
$$

where $y=Q^{2} / s x$ is the inelasticity variable. Therefore, the structure function $F_{L}$ is an auxiliary observable to detect saturation effects (unitarity corrections) in the gluon distribution.

Experimentally, the determination of the $F_{L}$ is quite limited, providing few data points. Most recently, the H1 Collaboration has determined the longitudinal structure function through the reduced double differential cross section [30],

$$
\sigma_{r} \equiv F_{2}\left(x, Q^{2}\right)-\frac{y^{2}}{Y_{+}} \cdot F_{L}\left(x, Q^{2}\right)
$$

where $Y_{+}=1+(1-y)^{2}$. For large inelasticity, the reduced cross section becomes $\left(F_{2}-F_{L}\right)$ and the contribution of $F_{L}$ is enhanced for large $y$. The longitudinal structure function should be obtained only in the region of large inelasticity, covered in a large range at HERA. In Ref. [30], two methods were used to perform the extraction: (i) for large $Q^{2}$ $>10 \mathrm{GeV}^{2}, \quad F_{L}$ is obtained through the extrapolation method, using a NLO DGLAP QCD fit (in the restricted kinematic range $y<0.35$ and $Q^{2} \leqslant 3.5 \mathrm{GeV}^{2}$ ) to extrapolate $F_{2}$ into the high $y$ region. (ii) At low $Q^{2}<10 \mathrm{GeV}^{2}$, the behavior of $F_{2}$ as a function of $\ln y$ is obtained using the derivative method, based on the cross section derivative $\left(\partial \sigma_{r} / \partial \ln y\right)_{Q^{2}}$. The data points obtained are consistent with the previous measurements; however, they are more precise and lie in a broader kinematical range. Therefore, in the following we use only the new data points to analyze.
In Fig. 6 we present the estimates for the $F_{L}$ structure function, in representative virtualities as a function of $x$. For the calculations, it was considered light quarks $(u, d, s)$ with effective mass $m_{q}=300 \mathrm{MeV}$ and the target radius $R_{A}^{2}$ $=5 \mathrm{GeV}^{-2}$. The large $r$ region is considered by the freezing of the gluon distribution at that region. Our expression for the observable is then

$$
F_{L}\left(x, Q^{2}\right)=\frac{Q^{2}}{4 \pi^{2} \alpha_{\text {em }}} \int_{0}^{\infty} d^{2} \mathbf{r} \int_{0}^{1} d z\left|\Psi_{L}(z, \mathbf{r})\right|^{2} \sigma_{\text {dipole }}^{G M}\left(x, \mathbf{r}^{2}\right) .
$$

The behavior is in agreement with the experimental result, each in shape as in normalization. A better description can be obtained by fine tuning the target size or the considered gluon distribution function; however, it should be stressed that the present prediction is parameter-free and determined using the dipole picture taking into account unitarity (saturation) effects in the effective dipole cross section. We verify that the rest frame calculation, taking into account the dipole degrees of freedom and unitarity effects, produces similar conclusions to those ones using the Breit system. For instance, in a previous work [13], the unitarity corrections to the longitudinal structure function were estimated in the laboratory frame considering Eq. (24), with unitarized expressions for $F_{2}$ and $x G\left(x, Q^{2}\right)$, obtaining that the expected corrections reach $70 \%$ as $\ln (1 / x)=15$, namely on the kinematical sector of the upcoming THERA project.

The higher twist corrections to the longitudinal structure function have been pointed out. For instance, Bartels et al. [8] have calculated numerically the twist-four corrections and found that they are large for $F_{T}$ and $F_{L}$, although with opposite signs. This fact leads from the remaining small effects to the inclusive structure function by almost a complete 
cancellation between those contributions. The higher twist content is analyzed considering the model [23] as an initial condition.

Concerning $F_{L}$, it was found that the twist-four correction is large and has negative signal, concluding that a leading twist analysis of $F_{L}$ is unreliable for high $Q^{2}$ and not too small $x$. The results are in agreement with the simple parametrization for higher twist (HT) studied by the Martin, Roberts, Stirling, and Thorne (MRST) in Ref. [31], where $F_{2}^{H T}\left(x, Q^{2}\right)=F_{2}^{L T}\left(x, Q^{2}\right)\left\{1+\left[D_{2}^{H T}(x) / Q^{2}\right]\right\}$. The second term would parametrize the higher twist content. In our case, the unitarity corrections provide an important amount of higher twist content; namely, it takes into account some of the several graphs determining the twist expansion (for recent discussions in these issues, see [32]).

\section{The charm structure function $F_{2}^{c \bar{c}}\left(x, Q^{2}\right)$}

In perturbative $\mathrm{QCD}$, the heavy quark production in the electron-proton interaction occurs basically through photongluon fusion, in which the emitted photon interacts with a gluon from the proton generating a quark-antiquark pair. Therefore, the heavy quark production allows us to determine the gluon distribution and the amount of unitarity (saturation) effects for the observable. In particular, charmed mesons have been measured at deep inelastic at HERA and the corresponding structure function $F_{2}^{c \bar{c}}\left(x, Q^{2}\right)$ is defined from the differential cross section for the $c \bar{c}$ pair production,

$$
\frac{d^{2} \sigma^{c \bar{c}}}{d x d Q^{2}}=\frac{2 \pi \alpha_{\mathrm{em}}}{x Q^{4}}\left[1+(1-y)^{2}\right] F_{2}^{c \bar{c}}\left(x, Q^{2}\right),
$$

with $y$ being the inelasticity variable. In the laboratory frame, the dominant mechanism is the boson-gluon fusion $\gamma^{*} G$ $\rightarrow c \bar{c}$. Hence, the charm structure function is directly driven by the gluon distribution and provides constraints for the gluonic function. In leading order (LO), it is written as [13]

$$
F_{2}\left(x, Q^{2}, m_{c}^{2}\right)=\frac{4 \alpha_{s}\left(\mu_{F}^{2}\right)}{9 \pi} \int_{a_{c} x}^{1} \frac{d y}{y} C_{g, 2}^{c}\left(\frac{x}{y}, \frac{m_{c}^{2}}{Q^{2}}\right) x G\left(y, \mu_{F}^{2}\right),
$$

where $a_{c}=\left[1+\left(m_{c}^{2} / Q^{2}\right)\right]$. The mass factorization scale lies in the range $m_{c}^{2} \leqslant \mu_{F}^{2} \leqslant 4\left(Q^{2}+4 m_{c}^{2}\right)$. Such a scale introduces an uncertainty of about $10 \%$, and an additional source of uncertainty is the charm mass, in general ranging on 1.2 $\leqslant m_{c} \leqslant 1.7 \mathrm{GeV}$. The standard QCD coefficient function is labeled by $C_{g, 2}^{c}\left(z, m_{c}^{2} / Q^{2}\right)$.

Experimentally, the latest measurements of the charm structure function are obtained by measuring mesons $D^{* \pm}$ production [33]. From the theoretical input, it was used as NLO coefficient functions, considering charm mass $m_{c}$ $=1.4 \mathrm{GeV}$ and the factorization-normalization scale $\mu_{F}$ $=\sqrt{Q^{2}+4 m_{c}^{2}}$. The function $F_{2}^{c \bar{c}}\left(x, Q^{2}\right)$ shows an increase with decreasing $x$ at constant values of $Q^{2}$, whereas the rise becomes more intense at higher virtualities. The data are consistent with the NLO DGLAP calculations. Concerning the ratio $R^{c \bar{c}}=F_{2}^{c \bar{c}} / F_{2}$, the charm contribution to $F_{2}$ grows steeply as $x$ diminishes. It contributes less than $10 \%$ at low $Q^{2}$ and reaches to about $30 \%$ for $Q^{2}>120 \mathrm{GeV}^{2}$ [33].

Once more the color dipole picture will provide quite a simple description for the charm structure function in a factorized way. Now, the Glauber-Mueller dipole cross section is weighted by the photon wave function constituted by a $c \bar{c}$ pair with mass $m_{c}$. Our expression for the charmed contribution in deep inelastic is thus written as

$$
\begin{aligned}
F_{2}^{c \bar{c}}\left(x, Q^{2}\right)= & \frac{Q^{2}}{4 \pi^{2} \alpha_{\mathrm{em}}} \int_{0}^{\infty} d^{2} \mathbf{r} \int_{0}^{1} d z \\
& \times\left[\left|\Psi_{T}^{c \bar{c}}(z, \mathbf{r})\right|^{2}+\left|\Psi_{L}^{c \bar{c}}(z, \mathbf{r})\right|^{2}\right] \\
& \times \sigma_{\text {dipole }}^{G M}\left(x, \mathbf{r}^{2}\right),
\end{aligned}
$$

where $\left|\Psi_{T, L}^{c \bar{c}}(z, \mathbf{r})\right|^{2}$ is the probability of finding in the photon the $c \bar{c}$ color dipole with the charmed quark carrying fraction $z$ of the photon's light-cone momentum with $T, L$ polarizations. For the correspondent wave functions, the quark mass in Eqs. (2), (3) should be substituted by the charm quark mass $m_{c}$. Here, we should take care of the connection between the Regge parameter $x=\left(W^{2}+Q^{2}\right) /\left(Q^{2}+4 m_{q}^{2}\right)$ and the Bjorken variable $x_{\mathrm{Bj}}$. For calculations with the light quarks these variables are equivalent; however, for heavier quarks the correct relation is [29]

$$
x_{\mathrm{Bj}}=x\left(\frac{Q^{2}}{Q^{2}+4 m_{c}^{2}}\right) \text {. }
$$

In Fig. 7 we show the estimates for the charm structure function as a function of $x_{\mathrm{Bj}}$ [Eq. (30)] for representative virtualities. In our calculations, the charm mass used was $m_{c}=1.5 \mathrm{GeV}$, the target size $R_{A}^{2}=5 \mathrm{GeV}^{-2}$, and the frozen gluon distribution at large $r$. We have verified a small soft contribution as in the $F_{L}$ case, decreasing as the virtuality rises. There is a slight sensitivity to the value of the charm mass, increasing the overall normalization as $m_{c}$ diminishes. Such a feature suggests that the charm mass is a hard scale suppressing the nonperturbative contribution to the corresponding cross section, which is in agreement with the recent Balitski1-Fadin-Kuraev-Lipatov (BFKL) color dipole calculations of Nikolaev and Zoller [29] and Donnachie and Dosch [22].

Regarding the Breit system description, in Ref. [13] strong corrections were found to the charm structure function, which are larger than those of the $F_{2}$ ones making use of expression (28). Considering the ratio $R_{2}^{c}$ $=F_{2}^{c \mathrm{GM}}\left(x, Q^{2}\right) / F_{2}^{c \operatorname{DGLAP}}\left(x, Q^{2}\right)$, the corrections predicted by the Glauber-Mueller approach would reach $62 \%$ at values of $\ln (1 / x) \approx 15$ (THERA region). Then, an important result is a large deviation of the standard DGLAP expectations at small $x$ for the ratio $R^{c \bar{c}}=F_{2}^{c \bar{c}} / F_{2}$ due to the saturation phenomena (unitarization). With our calculation one verifies that 


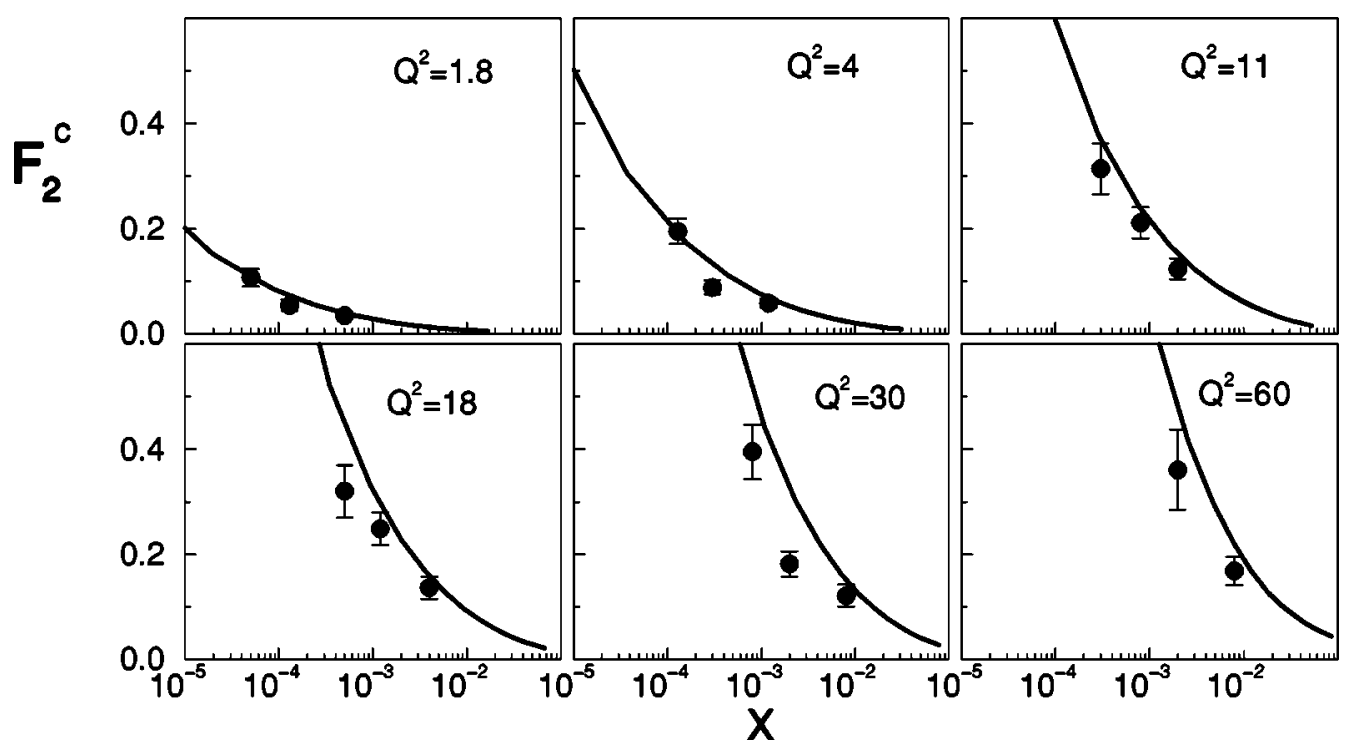

FIG. 7. The Glauber-Mueller result for the $F_{2}^{c \bar{c}}$ structure function as a function of the Bjorken variable $x$ at fixed virtualities (in GeV ${ }^{2}$ ). One uses charm mass $m_{c}=1.5 \mathrm{GeV}$, target size $R_{A}^{2}=5 \mathrm{GeV}^{-2}$, and frozen gluon distribution at large $r$. Data are from the ZEUS Collaboration [33] (statistical errors only).

a good description was obtained of data in both reference systems, suggesting a consistent estimation of the unitarity effects for this quantity.

\section{CONCLUSIONS}

We study the dipole picture for the description of deep inelastic scattering, focusing on observables driven directly by the gluon distribution. Starting from the dipole cross section provided by the Glauber-Mueller approach in QCD, we perform estimates for the inclusive structure function $F_{2}$, the longitudinal function $F_{L}$, and the charm structure function on the proton $F_{2}^{c \bar{c}}$.

For each of the observables discussed, we obtain theoretical estimates, in the rest frame, without a further fitting procedure, in good agreement with the updated data from HERA. The resulting calculations corroborate a quite consistent picture for the unitarity corrections from the GlauberMueller approach in both Breit and the rest reference systems. In the laboratory frame the unitarity effects are connected with the gluon distribution function, whereas in the color dipole framework the basic block is the dipole cross section which is corrected considering saturation effects.

The small transverse separation $r$ region is dominated by the leading logarithmic DGLAP formalism, with the additional ingredient of the unitarization phenomenon as the momentum fraction acquires quite small values. Such corrections are associated with the taming of the gluon distribution in the very small $x$ region, in general named the saturation regime. However, it should be stressed that the GlauberMueller approach and similar eikonal-like models provide a logarithmic $\ln (1 / x)$ asymptotic behavior for the inclusive structure function and gluon distribution, instead of a constant value for asymptotic energies.

The large transverse separation is described by nonpertur- bative aspects of QCD. Since this domain is not well determined at the moment, some modelling of the soft region is needed. In this work we choose the Ansatz in which the gluon distribution is frozen for virtualities above a cut radius $r^{2}>r_{\text {cut }}^{2}$, which corresponds to the region $Q^{2}<Q_{0}^{2}$. A convenient choice for the gluon PDF in order to cover the widest possible kinematical window diminishes the uncertainty coming from the soft sector. The most appropriated input is the GRV94 parametrization, where $r_{\text {cut }}=0.6 \mathrm{fm}$ is found, whereas it can take values $r_{\text {cut }}=0.4-0.5 \mathrm{fm}$ for the more recent PDF's. Throughout the paper we used the target size $R_{A}^{2}=5 \mathrm{GeV}^{-2}$, which corresponds to strong unitarity corrections.

When performing a comparison with the phenomenological model GBW, we have found that the Glauber-Mueller approach underestimates the dipole cross section from GBW at not small $x \geqslant 10^{-3}$. Instead, for very low $x \leqslant 10^{-4}$ the Glauber-Mueller approach overestimates the GBW model due to the strong increase of the gluon function in this region. Concerning the saturation model with DGLAP evolution (BGK), it is a particular case of the GM approach when considering central scattering $b=0$. Despite that the BGK model matches DGLAP evolution, the Glauber-Mueller approach describes more properly the realistic impact parameter dependence of the process. Moreover, in the GM approach the extension to the nuclear case is built in.

When considering the structure function $F_{2}$, we have found that it is dominated by small transverse distance contributions. However, a non-negligible content from the soft sector is present. Moreover, the photon wave functions enhance the dipole cross section into smaller dipole sizes, since the weight function selects smaller $r$ as the virtuality $Q^{2}$ diminishes. Our estimates here are parameter-free; however, a fine tuning of the parameters can improve the data description. Furthermore, we notice that in calculations from [10], 
only the aligned jet dipole configuration $z,(1-z) \approx 0$ (and the only transverse contribution) is considered, whereas we take into account all configurations, including the symmetric ones. Thus, all dipole sizes, even those from the nonperturbative region, are included in our results.

Concerning $F_{L}$, the estimates are consistent with the previous calculations in the Breit system and are in good agreement with data. A remarkable feature is that the GlauberMueller approach in the color dipole framework gives important higher twist contributions to the leading twist calculation in a simple way. As is well known, $F_{L}$ is the main quantity to study the expected higher twist effects in low virtualities.

The function $F_{2}^{c \bar{c}}$ is directly dependent on the gluon distribution and important unitarity corrections had been found when considering the Breit frame. Here, we verify consistent results in the rest frame in comparison with the previous ones in the fast proton system. We verified that the charm mass suppressed soft contributions in comparison with the $F_{2}$ case, and the results present a slight dependence with the specific value of $m_{c}$.

In conclusion, the Glauber-Mueller approach provides a well established formalism to take into account the unitarity effects. It allows us to estimate the higher twist contributions to relevant observables in a simplified way, while matching DGLAP evolution equation at Born level and including the impact parameter dependence properly.

\section{ACKNOWLEDGMENTS}

M. V. T. M. acknowledges Martin McDermott (Liverpool University-UK), Igor Ivanov (IKP-Forschungszentrum Juelich, Germany), and Victor Gonçalves (IFM-UFPel, Brazil) for useful enlightenments. This work was partially financed by $\mathrm{CNPq}$, Brazil.
[1] Yu.L. Dokshitzer, Sov. Phys. JETP 46, 641 (1977); G. Altarelli and G. Parisi, Nucl. Phys. B126, 298 (1977); V.N. Gribov and L.N. Lipatov, Sov. J. Nucl. Phys. 28, 822 (1978).

[2] M.B. Gay Ducati, Braz. J. Phys. 31, 115 (2001).

[3] G.R. Kerley and M. McDermott, J. Phys. G 26, 683 (2000); M.F. McDermott, hep-ph/0008260.

[4] V.N. Gribov, Sov. Phys. JETP 29, 483 (1969).

[5] N.N. Nikolaev and B.G. Zakharov, Z. Phys. C 49, 607 (1991); Phys. Lett. B 260, 414 (1991); Z. Phys. C 53, 331 (1992).

[6] M. Wüsthoff, Phys. Rev. D 56, 4311 (1997).

[7] E. Gotsman, et al., J. Phys. G 27, 2297 (2001).

[8] J. Bartels, K. Golec-Biernat, and K. Peters, Eur. Phys. J. C 17, 121 (2000).

[9] A.H. Mueller, Nucl. Phys. B335, 115 (1990).

[10] A.L. Ayala, M.B. Gay Ducati, and E.M. Levin, Nucl. Phys. B493, 305 (1997); B511, 355 (1998).

[11] L.V. Gribov, E.M. Levin, and M.G. Ryskin, Phys. Rep. 100, 1 (1983).

[12] A.L. Ayala, M.B. Gay Ducati, and E.M. Levin, Eur. Phys. J. C 8, 115 (1999).

[13] A.L. Ayala, M.B. Gay Ducati, and V.P. Gonçalves, Phys. Rev. D 59, 054010 (1999).

[14] M.B. Gay Ducati and V.P. Gonçalves, Phys. Lett. B 487, 110 (2000); 491, 375(E) (2000).

[15] M.B. Gay Ducati and V.P. Gonçalves, Phys. Rev. C 60, 058201 (1999); M.B. Gay Ducati and V.P. Gonçalves, Phys. Lett. B 466, 375 (1999); V.P. Gonçalves, ibid. 495, 303 (2000).

[16] M.B. Gay Ducati and V.P. Gonçalves, Nucl. Phys. B (Proc. Suppl.) 79, 302 (1999); Nucl. Phys. B557, 296 (1999).

[17] A.L. Ayala, M.B. Gay Ducati, and E.M. Levin, Phys. Lett. B
388, 188 (1996); M.B. Gay Ducati and V.P. Gonçalves, ibid. 502, 92 (2001).

[18] E. Gotsman et al., Phys. Lett. B 492, 47 (2000); Nucl. Phys. A683, 383 (2001); Phys. Lett. B 506, 289 (2001).

[19] V. Del Duca, S.J. Brodsky, and P. Hoyer, Phys. Rev. D 46, 931 (1992).

[20] J.R. Forshaw and D.A. Ross, QCD and the Pomeron (Cambridge University Press, Cambridge, England, 1997).

[21] I.P. Ivanov and N.N. Nikolaev, Phys. At. Nucl. 64, 753 (2001).

[22] A. Donnachie and H.G. Dosch, Phys. Lett. B 502, 74 (2001); Phys. Rev. D 65, 014019 (2002).

[23] K. Golec-Biernat and M. Wüsthoff, Phys. Rev. D 59, 014017 (1999); Phys. Rev. D 60, 114023 (1999).

[24] M. McDermott, L. Frankfurt, V. Guzey, and M. Strikman, Eur. Phys. J. C 16, 641 (2000).

[25] E. Gotsman, E. Levin, and U. Maor, Nucl. Phys. B493, 354 (1997).

[26] A. Kovner and U.A. Wiedemann, Phys. Rev. D 64, 114002 (2001).

[27] M. Glück, E. Reya, and A. Vogt, Z. Phys. C 67, 433 (1995).

[28] J. Bartels, K. Golec-Biernat, and H. Kowalski, hep-ph/0203258.

[29] N.N. Nikolaev and V.R. Zoller, Phys. Lett. B 509, 283 (2001).

[30] H1 Collaboration, C. Adloff et al., Eur. Phys. J. C 21, 33 (2001).

[31] A.D. Martin, R.G. Roberts, W.J. Stirling, and R.S. Thorne, Phys. Lett. B 443, 301 (1998).

[32] E. Gotsman et al., Phys. Lett. B 506, 289 (2001); Nucl. Phys. A683, 283 (2001).

[33] ZEUS Collaboration, S. Breitweg et al., Eur. Phys. J. C 12, 35 (2000). 